\title{
LO SPIRITO MANIFESTATORE, NELLA PNEUMATOLOGIA DI PAVEL N. EVDOKIMOV
}

\author{
The Manifesting Spirit in the Pneumatology of Pavel N. Evdokimov
}

Alberta Maria Putti *

SOMMARIO THEMATICO: L'uomo nuovo, l'uomo pneumatico, è al centro della riflessione teologica di Pavel N. Evdokimov (1901-1970). Argomenta che l'azione dello Spirito Santo non tanto è comprensibile con una analisi esclusivamente oggettiva dell'agire di Dio in relazione all'uomo. Egli ritiene si tratti di riconoscere l'importanza dell'accoglienza dei doni dello Spirito dentro un duplice movimento: l'uomo vive prima una fase di conversione, e di lotta, a cui segue un movimento di ascesi che scaturisce dall'aver accolto i doni dello Spirito Santo. Santificandoci, lo Spirito Santo trasmette "qualcosa" della sua propria natura perché noi si possa compiere la volontà del Padre. Lo Spirito Santo imprime nell'uomo il suo Soffio vitale e, senza confusione, si fa co-soggetto della nostra vita in Cristo. L'uomo che è stato risoffiato dal Figlio del suo stesso Spirito, diventa immagine di Cristo nel mondo; respira del suo stesso soffio, della sua stessa vita.

PAROLE CHIAVI: Eudokimov. Pneumatologia. Antropologia pneumatica. Cristologia. Spirito Santo.

\begin{abstract}
The new, pneumatic, person is central to Pavel N. Evdokimov's (1901-1970) theological work. He argues that the Holy Spirit's action is not understandable with an exclusively objective analysis of God's action in relationship to humanity. Rather, the human person welcomes the gifts of the Holy Spirit within a double movement: the first phase is that of conversion and struggle, after which there is a phase of generous asceticism that springs forth from having welcomed the Holy Spirit's gifts. In sanctifying us, the Holy Spirit communicates something that is proper to the Spirit's nature, enabling us to complete the Father's will. The
\end{abstract}

\footnotetext{
* Pontificia Università Gregoriana, Roma, Itália.
} 
Holy Spirit imparts to us His life-giving breath so that, without confusion, the Holy Spirit becomes co-subject of our life in Christ. The human person who has received this new life becomes an image of Christ in the world, breathing with his very breath, living his very life.

KEYWORDS: Evdokimov. Pneumatology. Pneumatic Anthropology. Pneumatic Christology. Holy Spirit.

\section{Introduzione}

T'uomo nuovo, l'uomo pneumatico, "signore onnipotente del suo des¿tino e della sua storia" (EVDOKIMOV, 2015, p. 55), è al centro della riflessione teologica di Pavel Nikolaevič Evdokimov, teologo e filosofo russo (1901-1970) grande conoscitore del pensiero ortodosso, interprete e testimone della teologia orientale nel vasto orizzonte culturale dell'Europa del secolo scorso. Il suo contributo maggiore fu certamente quello di aver fatto conoscere nel mondo occidentale la corrente del pensiero filosofico-teologico di Vladimir S. Solov'ëv, Sergej N. Bulgakov e Nikolaj Berdjaev sulla scia dei quali si era posto (ARJAKOVSKY, 2002, p. 604-610).

Nell'ambito della teologia occidentale Evdokimov divenne uno dei tramiti principali del pensiero teologico russo ${ }^{1}$, coniugando il discorso su Dio con l'accurata e viva attenzione alla condizione dell'uomo. La teologia ortodossa, che in modi diversi si era fatta strada nel panorama Europeo, in dialogo critico e a volte anche avverso, con le correnti culturali emergenti del ' $900^{2}$, portava in sé una forte visione pneumatologica ancorata alla tradizione dei Padri e nel contempo era protesa verso una lettura integrale del mistero del Dio Uno e Trino. Rispetto alla tradizione latina che aveva una pneumatologia di minor evidenza, frammentata nell'analisi sistematica della teologia di scuola, l'incontro con il pensiero russo fu certamente avviamento di nuove analisi. Vita e teologia sono congiunte nel teologare e ciò era chiaro agli occhi del nostro:

La vocazione teologica - scrive - invita a superare la sufficienza di una scienza puramente enciclopedica, poiché essa non è affare della ragione naturale, ma si radica nella luce del Verbo. Nella loro iniziazione, i Padri mostrano l'ascesi

\footnotetext{
${ }^{1}$ In questo contesto, fu certamente complice la situazione storica della diaspora Parigina: sullo sviluppo storico dell'apertura della cultura francese alla teologia russa vedi FOURCADE, M., De Soloviev à Boulgakov: la réception catholique de l'orthodoxie russe en France. Contacts, Paris, n. 238, p. 118-145, Avril-Juin. 2012. L'intero numero è dedicato alla Emigrazione russa e la cultura spirituale in Occidente. M. Guittat-Naudin, «La Réception de Valdimir Soloviev en France», Vladimir Soloviev, Jacques Maritain et le personnalisme chrétien, P. de Laubier, Presses universitaires de l'IPC/Parole et Silence, 2008. p. 75-98.

2 «L'emigrazione russa è chiamata a preservare la continuità della cultura spirituale russa e, al meglio delle sue capacità, a contribuire al suo sviluppo creativo»: (BERDJAEV, 1925, p. 5).
} 
come il preliminare dell'arte teologica e l'orazione come uno stato - katastasis - dell'intelligenza, una recettività orante, aperta alle rivelazioni sfolgoranti del Trascendente (EVDOKIMOV, 1971, p. 26).

La teologia latina si sentì provocata fino a proporre, dopo le istanze avvertite al Concilio Vaticano II (PUTTI, 2016a, p. 362-394), una sintesi sulla riflessione teologica riguardo allo Spirito Santo, di cui si fece portatore Y. Congar col suo Credo nello Spirito Santo (CONGAR, 1981). Egli, proprio partendo dalla ricchezza della tradizione condivisa da tutta la cristianità e rispettoso delle differenti sensibilità confessionali, stese la più importante opera di sintesi della teologia pneumatologica nella tradizione latina. Sicuramente il confronto con Evdokimov fu a tal riguardo un importante fase preparatoria di tale elaborazione ${ }^{3}$.

Una breve digressione riguardante lo sviluppo della teologia latina può ora giovare al nostro argomento. Quanto è stata influente la teologia orientale nel secolo scorso? La teologia arrivata al Concilio Vaticano II, e dibattuta attorno al tema pneumatologico, quanto è stata toccata e influenzata, o addirittura preparata, dalla teologia russa ${ }^{4}$ (EVDOKIMOV, 1965, p. 83.), oltre che più ampiamente dalla teologia orientale? La domanda nasce dal fatto che, seguendo lo sviluppo delle idee teologiche che si sono andate ordinando nella tradizione latina, si può individuare un graduale cambio di sensibilità.

Nel contesto pneumatologico vi fu un'autentica novità dell'analisi sistematica: la prospettiva squisitamente causale, che la neoscolastica aveva fortemente voluto saldare alla teologia della grazia, lasciava il passo ad una visione integrale, totale, ampliata a differenti ambiti, liberamente adesa ai contenuti della teologia trinitaria, antropologica ed ecclesiologica (CONGAR, 2011, p. 365-412). La visione pneumatologica sembra emergere con chiarezza come contesto unitario, o se vogliamo in senso diverso, come argomento di sintesi, che potremmo chiamare: teologia organica dello Spirito Santo $^{5}$. Quanto qui affermato è visibile nella continuità dei temi teologici

\footnotetext{
${ }^{3}$ Evdokimov l'autore orientale più citato da Y. Congar nella parte 3a del suo Credo nello Spirito Santo. «Lo Spirito Santo soltanto può trascendere le barriere senza relativizzare nulla, ma anzi completando e integrando tutti gli aspetti della fede cristiana nella loro pienezza ricca di infinite sfumature»: (EVDOKIMOV, 1981b, p. 501).

${ }^{4}$ La convinzione della necessità di un incontro di scambio e di maturazione della teologia cristiana emerge negli scritti di Evdokimov, il quale era convinto che l'evento storico dell'emigrazione Russa in Francia fosse provvidenziale, e che in esso si celasse un significato tutto da decifrare. A noi sembra che il senso più profondo di quell'evento si possa oggi valutare dall'esito del Concilio Vaticano II in cui, un confronto e di uno sviluppo congiunto della teologia dello Spirito Santo, ha portato nella tradizione latina ad un ripensamento del tema dello Spirito in teologia (EVDOKIMOV, 1965, p. 83).

${ }^{5} \mathrm{Se}$, come riteneva Evdokimov, la vocazione teologica invita a superare una visione puramente analitica e causalistica, ciò potrebbe far comprendere la ragione della strutturazione teologica che la tradizione latina ha avuto: quando la teologia latina ha considerato lo Spirito Santo come un tema riferibile solo agire causale di Dio nella vita dell'uomo, abbiamo perso il nesso tra conoscenza e vita, la teologia ha perso la sua funzione radicata nel cuore del teologo, per il proprio essere in Cristo mosso dallo Spirito Santo. Una teologia causalistica portava all'estremo di fissismi poiché separava la teologia dalla vita, l'intelligenza della fede dalla preghiera.
} 
che alcuni autori latini hanno aperto al contatto di correnti nuove. Si tratta di nomi noti che hanno influenzato la loro posterità. Louis Bouyer, Hans Urs von Balthasar, Jean Daniélou e Pierre Teilhard de Chardin subirono l'influsso della teologia russa, in particolar modo dialogarono col pensiero di S. Bulgakov (ARJAKOVSKY, 2002, p. 607). Questa digressione merita uno studio accurato a parte e sarà oggetto di analisi successive.

Nella lettura dei testi maggiori di Evdokimov, pubblicati nell'ultima parte della sua vita, risalta una prospettiva teologica peculiarmente unitaria in cui l'antropologia viene congiunta alla visione cristologica e pneumatologica: l'uomo che vive in Cristo è adombrato dallo Spirito.

Dal momento dell'epifania al Giordano "lo Spirito è visibile sull'umanità di Gesù e vi riposa".

Questo momento della vita del Figlio di Dio è paradigmatico: espressione e manifestazione della condizione del Figlio, che è partecipata ai figli; lo Spirito adombra coloro che partecipano, in forza del sacramento del battesimo, della condizione filiale. Scrive su questo Evdokimov:

Al momento dell'Epifania, lo Spirito, in modo a tutti visibile, discende sulla umanità di Gesù e vi riposa. Il giorno della Pentecoste egli discende nel mondo "in Persona", ipostaticamente, e diviene operante al di dentro della natura umana. Egli agisce, dunque, all'interno di noi, ci muove, ci rende dinamici, e santificandoci ci trasmette qualcosa della sua propria natura. Senza confusione, lo Spirito si identifica con noi, si fa co-soggetto della nostra vita in Cristo, più intimo a noi che noi stessi (EVDOKIMOV, 1971, p. 124).

Tratto da Lo Spirito nella tradizione Ortodossa questo brano costituisce il cardine della nostra analisi, così ordinato perché ci appare chiaro che l'autore abbia in esso voluto dipingere, in una sapiente armonia di riflessioni profonde, i punti focali della teologia dello Spirito Santo, per questo lo riprenderemo gradualmente, procedendo in modo argomentativo, sui contenuti di maggior rilievo.

\section{Al momento dell'Epifania, lo Spirito, discende sull'umanità di Gesù e vi riposa}

Lo Spirito Santo riposa sul Figlio al momento del suo Battesimo al Giordano.

E lo Spirito testimonia la divinità del Cristo: si presenta simbolicamente sopra Colui che gli è del tutto uguale. Una voce proviene dalle profondità dei cieli, da quelle stesse profondità dalle quali proveniva chi in quel momento riceveva la testimonianza. Lo Spirito appare visibilmente come colomba e, in questo modo, onora anche il corpo divinizzato e quindi Dio. Non va dimenticato che molto tempo prima era stata pure una colomba quella che aveva annunziato la fine del diluvio. Purificatevi totalmente e progredite in questa purezza. Dio di nessuna cosa tanto si rallegra, come della conversione e della salvezza 
dell'uomo. Per l'uomo, infatti, sono state pronunziate tutte le parole divine e per lui sono stati compiuti i misteri della rivelazione (Gregorio Nazianzeno, PG 36, p. 358-359).

Ciò che avviene nella storia della salvezza è espressione del movimento eterno di amore trinitario, che i Padri - come abbiamo letto dalle parole di Gregorio Nazianzeno - ritenevano esplicativo della manifestazione di Dio. L'eterno movimento di amore del Padre, del Figlio e dello Spirito Santo ha come fonte ipostatica la persona del Padre: il Figlio e lo Spirito sono congiunti nella manifestazione del Padre sebbene distinti come «due persone provenienti dallo stesso Padre. [...] lo Spirito riposa eternamente sul Figlio e lo manifesta»(EVDOKIMOV, 1971, p. 56). Lo Spirito Santo è, quindi, lo Spirito del Figlio; egli dimora nel Figlio e in coloro che gli appartengono, essendo il Tuttosanto, poiché è la santità stessa di Dio, nel suo nome esprime ciò che egli è, e che al contempo è a lui proprio ed è anche misterioso e nascosto. Egli è ritenuto il Dono ed il Donatore, proprio perché è colui che dispensa i doni. Egli stesso si dona: santifica, divinizza, vivifica, abita l'uomo, lo riempie dell'amore ( $R m$ 5,5) che è del Padre e del Figlio. Lo Spirito Santo realizza nell'uomo il suo vivere nella storia ad immagine di Dio: come il Figlio è stato uomo, Colui che era Dio, così l'uomo può vivere al modo di Dio $(\mathrm{Gv} 14,20)^{6}$.

Il Battesimo dell'acqua e dello Spirito quindi costituisce l'uomo in Cristo ad immagine del Figlio e, nell'Unzione, lo Spirito fa l'uomo somigliante.

Lo Spirito Santo che è stato dato dal Figlio dopo la sua ascensione, dal dimorare presso i discepoli, compie un movimento nell'essere in loro: "perché egli dimora presso di voi e sarà in voi" (Gv 14,17), dal momento della pentecoste lo Spirito abiterà il cuore dell'uomo lo introdurrà nel Regno (EVDOKIMOV, 1971, p. 114). Inizia così un movimento continuo, sistolico-diastolico, che è insieme dello Spirito e dell'uomo ${ }^{7}$, il quale nella libertà partecipa e si conforma al volere del Padre. Sempre più somiglianti, sempre più ad immagine e somiglianza della visione del Padre, i cristiani manifestano d'essere figli, poiché vivono la creazione nuova che è la condizione dei salvati ${ }^{8}$.

${ }^{6}$ Così Yannaras chiude l'argomento sopra introdotto: «L'unità teantropica dell'uomo al corpo di Cristo è un radicamento di sé nell'Altro. Se il tropos divino è l'amore: la vita l'uno nell'altro, per l'uomo il vivere in comune esprime il modo di esistenza che gli è proprio»: (YANNARAS, 1995, p. 41-42).

${ }^{7}$ La creazione e lo Spirito sono congiunti, nel senso che il mondo creato agisce-con lo Spirito, si tratta di una sinergia che possiamo anche chiamare co-azione: «Il mondo non può contenere la forza dello Spirito Santo nella sua pienezza senza fondersi ed essere da essa consumato. Nella kenosis (dello Spirito Santo) della discesa nel mondo, questa forza, autolimitandosi, è come se si indebolisse: il sinergismo tra la grazia e la libertà creaturale conduce ad una tale autolimitazione»: (BULGAKOV, 1991, p. 580).

8 «Si intende per creazione la trasformazione in meglio, che avviene quaggiù, di coloro che sono caduti in peccato - anche così si intende infatti secondo la forma espressiva consueta della Scrittura, come quando Paolo dice: "Se uno è in Cristo è una creatura nuova" - il rinnovamento che avviene, e il cambiamento di questa vita terrestre e passibile nella cittadinanza celeste, che ci viene per lo Spirito, questo innalza le nostre anime al massimo dello stupore»: (BASILIO DI CESAREA, 1993, p. 158). 
Nel processo di rivelazione del Figlio, lo Spirito Santo rivela l'uomo all'uomo, il quale così fa esperienza del proprio essere di Cristo ed in Cristo: «Io sono io, me stesso; colui che si pensa in se stesso e colui che si pensa nell'uomo, nella coscienza creata e teandrica; il Nome indicibile in me stesso, il Nulla apofatico e il Nome pronunziato: Ecce Homo, dimora Trina» (EVDOKIMOV, 1981b, p. 452-453).

È in questa condizione del suo essere creato in Cristo, e nella sua morte e risurrezione rigenerato alla vita nuova, che l'uomo comprende il senso della storia della salvezza: dall'incarnazione alla risurrezione, alla pentecoste. La chiave interpretativa della storia della salvezza va colta in Dio stesso il quale da sempre ha voluto divenire uomo e fare dell'umanità una teofania: “Dio ha creato il mondo per divenirvi uomo e perché l'uomo vi diventi dio per la sua grazia e partecipando alle condizioni dell'esistenza divina" (Atanasio). Queste parole scelte da Evdokimov vogliono proprio accentuare la relazione esistente tra creazione, incarnazione e redenzione, tutte vanno lette a partire da Dio, il quale vuole l'incarnazione per gli uomini e per la loro salvezza9 ${ }^{9}$ (EVDOKIMOV, 1981b, p. 84-85), focalizzandosi quindi sull'aspetto manifestativo di Dio che si rivela nell'incarnazione, e mostrando all'uomo il suo volto filiale, perché egli possa vivere in modo sacerdotale, partecipando alla Liturgia eterna che tutto di Cristo ci dona e tutto in lui ci fa donare.

Gesù chiede e riceve il battesimo di Giovanni Battista, battesimo di penitenza e di conversione, che era il segno del ravvedimento per coloro che lo chiedevano, ed era inteso come un lavacro di purificazione. Gesù accoglie di essere trattato come uno dei peccatori: nasconde la sua gloria divina e per restituire all'uomo la sua identità filiale sceglie una condizione di umiliazione (GIOVANNI CRISOSTOMO, PG 62, p. 219-229). La sua volontà sembra incomprensibile al Battista, ma la volontà sua è divina e umana ${ }^{10}(\mathrm{DH}, \mathrm{n}$. 556-558), è un continuo abbassamento verso l'umanità (GREGORIO DI NAZIANZO, PG 36, 100; PG 36, 285; ATANASIO, PG 25; CIRILLO DI ALESSANDRIA, PG 75; MASSIMO IL CONFESSORE, PG 91, XXXVIII, 28) per rendere gloria al Padre (ILARIO DI POITIERS, PL 10, 270; AGOSTINO DI IPPONA, PL 38, 1002-1003). Per questo, nella sua vita terrena, egli rivolse al Padre ogni suo atto di obbedienza (Gv 6, 38) fin sulla croce: divinità e umanità sono unite in Cristo indivisibilmente e assunte nella Trinità (PUTTI, 2016b, p. 1143-1146).

\footnotetext{
${ }^{9}$ Il riferimento teologico a cui torna Evdokimov è l'opera di S.N. Bulgakov L'Agnello di Dio. ${ }^{10}$ Cfr. Concilio di Calcedonia, Simbolo di fede: «un solo e medesimo Figlio, il Signore nostro Gesù Cristo, perfetto nella sua divinità e perfetto nella sua umanità, [...] uno e medesimo Cristo Signore unigenito, da riconoscersi in due nature, senza confusione, immutabili, indivise, inseparabili, non essendo venuta meno la differenza delle nature a causa della loro unione, ma essendo stata, anzi, salvaguardata la proprietà di ciascuna natura, e concorrendo a formare una sola persona e ipostasi»: (DH, n. 301-302).
} 
Ciò che avviene nel momento del Battesimo al Giordano è un evento epifanico che manifesta chi sia Gesù di Nazareth: il Cristo, l'Unto di Spirito Santo, che il Padre chiama "il Figlio amato" (Lc 3,22). Gesù, il Figlio Unigenito è riconosciuto come l'amato, in quel momento tutta la Trinità è rivelata ed al contempo, si preannuncia l'abbassamento del Figlio il quale vivrà una spoliazione sempre più esplicita nella storia fino alla morte di croce; la Trinità tutta, nel suo rivelarsi, appare coinvolta in un progressivo abbassamento, in cui sia il Padre che lo Spirito partecipano (BULGAKOV, 190, p. 13-27; EVDOKIMOV, 1983, p. 27-33; ID., 1981a, p. 101-105) alla kenosi del Figlio, dalla creazione all'incarnazione.

\section{A pentecoste lo Spirito discende "in persona", ipostaticamente e diviene operante al di dentro della natura umana}

Il Battesimo di Gesù è l'evento in cui si compie la rivelazione trinitaria: le persone divine si mostrano nel loro essere in relazione. " della Trinità s'irradia progressivamente" (GREGORIO DI NAZIANZO, Orationes, Or. 31, 26-27: PG 36, 161). Il Figlio viene in nome del Padre per farlo conoscere e compiere la sua volontà; lo Spirto viene in nome del Figlio per rendergli testimonianza, per manifestarlo e completare dei suoi doni l'opera del Cristo» (EVDOKIMOV, 1971, p. 112).

A riguardo del rivelarsi dello Spirito nella storia della salvezza, Evdokimov riprende primariamente la teologia di Sergej N. Bulgakov ${ }^{11}$ il quale riteneva che le controversie corse tra i teologi d'Oriente e d'Occidente, che per secoli si erano scontrati sulla questione della processione dello Spirito Santo, avessero distolto l'attenzione dal vero interesse che avrebbe dovuto animare la teologia sull'identità dello Spirito. Una "falsa problematica" riteneva avesse distolto dal bene maggiore: il rivelarsi del Padre nel Figlio per lo Spirito Santo (BULGAKOV, 2012, p. 281) affinché l'uomo divenisse il luogo della manifestazione della gloria di Dio.

Nel suo auto-rivelarsi, infatti, il Padre si manifesta immediatamente al Figlio il quale è l'immagine della sua ipostasi; il Figlio è manifestato nello Spirito Santo vivificante, ed è in tal senso che, secondo Atanasio a cui fa eco Bulgakov, lo Spirito Santo è l'immagine del Figlio.

Al momento dell'Epifania lo Spirito discende dal cielo come Colomba e riposa su Gesù. Nelle sue manifestazioni è movimento "verso Gesù", al fine di renderlo

11 «Dio è spirito. La spiritualità è l'essenza di Dio stesso. La spiritualità dello Spirito Santo, quale Terza ipostasi, è identica alla spiritualità di Dio, ma nello stesso tempo è da essa distinta ipostaticamente. Dio è spirito in tutto il suo essere ipostatico. Ma è lo Spirito Santo che manifesta nella Trinità la spiritualità di questa, come Dio Tri-ipostatico»: (BULGAKOV, 2012, p. 259). 
visibile e manifesto. La sua prescienza è nascosta nel Figlio come il soffio e la voce, che si fanno da parte di fronte alla parola che essi rendono udibile. Se il Figlio è l'immagine del Padre, lo Spirito Santo l'immagine del Figlio, lo Spirito, dicono i Padri, è il solo a non avere la sua immagine in un'altra Persona, egli è essenzialmente misterioso (EVDOKIMOV, 1971, p. 115).

Si tratta quindi di una volontà manifestativa delle persone divine le quali si rivelano reciprocamente, trinitariamente, relazionalmente: il Padre, per Filium, nello Spirito Santo - oppure - il Padre, per Spiritum Sanctum nel Figlio $^{12}$.

Ogni persona divina è una donazione sussistente nella circumsessione dei tre. Ciascuna è verso l'Altra, è il co-esse: la Persona è per la comunione tri-una, è essenzialmente da essa. A rigor di termini, è solo in Dio che la persona esiste. L'uomo, "immagine", ha nostalgia di diventare "persona" e non realizza questa vicinanza che nella partecipazione ad un Archetipo, totalmente teso verso l'Altro divino (EVDOKIMOV, 2001, p. 198-199).

E proprio per l'essere stato creato ad immagine di Dio che l'uomo riceve tutto dall'evento dell'incarnazione: vede l'icona, l'uomo perfetto, contempla la deiformità, Cristo che è divino umano.

Nel centro della sua persona l'uomo sperimenta il proprio sé, nel suo cuore - luogo della sua totalità - percepisce d'essere orientato al Cristo, calamitato dalla sua persona divina. La creatura umana si compie quando vive nella comunione con Dio assaporando a pieno la sua immagine: uomo di spirito, anima e corpo costituito. Così superando la dimensione solo umana, e in questo insieme spirituale e psico-somatica, tende verso Dio fino a trascendere: «la persona in questo senso si costruisce superandosi ed è in questo aspetto che esprime il termine "ipostasi", fondamentale per il destino di ogni essere umano»(EVDOKIMOV, 2001, p. 200).

Se quindi il destino dell'essere umano è la theosis - deificazione, l'uomo diviene persona in un processo continuo di attuazione di sé nella relazione con Dio e questo avviene tramite l'acquisizione della grazia, tramite i doni dello Spirito Santo. A tal riguardo Evdokimov prende una posizione chiara nel distinguere coloro che hanno ricevuto il battesimo da coloro che non lo hanno ricevuto. Non si sofferma sulla condizione di vita dei non battezzati, poiché è consapevole che essi sono affidati a Dio, poiché egli soltanto può esprimere un giudizio sul cuore dell'uomo. Mentre riflette sullo statuto di vita nuova di coloro che hanno ricevuto il battesimo: il battezzato è passato ad una condizione nuova di vita, allo statuto ontologico di nuova creatura, egli vive cioè il mistero della santità. L'uomo vecchio, naturale si contrappone all'uomo spirituale:

\footnotetext{
${ }^{12}$ Per questo il Figlio afferma nel vangelo di Giovanni (Gv 16): "Tutto quanto ha il Padre è mio (del Figlio)" e, ancora lo Spirito Santo "prenderà del mio" e "mi glorificherà".
} 
nello stato naturale l'uomo emerge nella sua condizione di individuo, isolato $^{13}$ (GIANAZZA, 1983, p. 60); soltanto quando lo Spirito Santo riposa su di lui allora l'identità dell'uomo è identità personale, identità per grazia, si costituisce dall'Alto per manifestare nella sua storia la propria identità filiale.

La somiglianza è l'ipostasi realizzata a cui l'uomo tende. Per questo egli può divenire sia somiglianza che dissomiglianza. L'uomo è dissomiglianza quando invece di realizzarsi come ipostasi divinizzata di un essere divinizzato nella sinergia con lo Spirito Santo, rimane individuo, vivendo soltanto come essere naturale (EVDOKIMOV, 2001, p. 199-202). Evdokimov rilegge qui la teologia di Atanasio e le sue conseguenze nella teologia russa per cogliere quale sia l'approfondimento dell'identità dello Spirito e del suo agire nell'uomo come manifestatore. Aspetto che prenderà dalla prospettiva antropologico-pneumatologica di S.N. Bulgakov.

Atanasio, per provare la divinità dello Spirito Santo, ha elaborato una teologia delle relazioni trinitarie; e, a partire dalla rivelazione neotestamentaria, volle affermare l'unità di operazione della Trinità, congiunta alla differenziazione delle persone, che tra loro sono inseparabili.

Le relazioni: Padre-Figlio e quella Figlio-Spirito sono propriamente diadiche e tra loro equilibrate. Infatti, le relazioni trinitarie possono essere lette specularmente: lo Spirito ha con il Figlio lo stesso tipo di rapporto che il Figlio ha con il Padre. Lo Spirito rispetto al Figlio è chiamato Spirito del Figlio (Gv 19, 30), al Figlio è unito, come è unito al Padre il Figlio. Lo Spirito Santo è inseparabile dal Verbo e non può essere mai considerato estraneo a lui. Egli ha tutto ciò che il Figlio gli dona, tutto ciò che lo Spirito ha, quindi, proviene dal Figlio. Per questo Atanasio dice che lo Spirito è immagine del Figlio, come il Figlio lo è del Padre. Allo stesso modo: "chi ha lo Spirito ha il Figlio, poiché lo Spirito è nel Figlio ed il Figlio è in lui" (Rm 8,9.11).

Lo Spirito è dato dal Figlio e glorifica il Figlio, così come il Figlio glorifica il Padre ed insieme allo Spirito lo glorifica. Dal Padre infatti lo Spirito procede ed è nel Padre: lo Spirito è unito alla divinità del Padre, perché è nel Verbo, il quale è nel Padre e se è vero che il Padre manda lo Spirito,

\footnotetext{
13 "L'uomo può dunque divenire "somiglianza" o "dissomiglianza" ma mai a-somiglianza. La sua stessa struttura deiforme rende impossibile una soluzione "autonoma" del suo destino umano. La sua autonomia si volge in "teonomia" o in "eteronimia": liberante la prima, asservente la seconda. Il termine stesso "persona" (prosopon), nel suo significato originario "di maschera", contiene un profondo messaggio: o è volto del tutto, icona di Dio, partecipazione all'essere; o è maschera del nulla, smorfia del demonio, partecipazione al non-essere. In altre parole "l'anima è il luogo delle presenze e degli incontri, la sua natura è coniugale: che sia fidanzamento o adulterio, essa è comunione con "altro". Non esiste una scelta tra angelismo e animalità, ma tra Dio e il demoniaco»: (EVDOKIMOV, 1981b, p. 124).
} 
ciò avviene nel Nome del Figlio: quando il Figlio "alitò" (Gv 20,22) sui discepoli era una creazione nuova. Questa visione che proponeva Atanasio, e che ha permeato la teologia patristica, coglie il senso di reciprocità delle persone divine nella Trinità: il Padre è tutto nel Figlio, che è sua immagine, il Figlio è tutto nel Padre, come suo proprio. Il Figlio è tutto nello Spirito come sua immagine e lo Spirito è tutto nel Figlio come suo proprio (CATTANEO, 1986, p. 20-26).

La teologia contemporanea converge nel ritenere che: «La cosa più interessante che si dice sullo Spirito è la sua appartenenza al Figlio, così che stabiliscono due relazioni parallele: Padre-Figlio, Figlio-Spirito Santo. Lo Spirito Santo è del Figlio come il Figlio è di Dio. In alcuni casi si dice che è proprio del Figlio o del Logos, ma anche di Dio (o del Padre)» (LADARIA, 2002, p. 234-235).

Come agisce lo Spirito, qual è la funzione che gli è propria nella vita dell'uomo? Ed in che modo il suo agire per l'Altro diventa nell'uomo un agire attraverso l'altro?

Nel discorso dell'ultima cena Giovanni descrive quale sia il ruolo dello Spirito Santo, Terza ipostasi: essere Colui che rivela un altro. Bulgakov riteneva che lo Spirito Santo, volendo restare nel mistero, si riveli e si nasconda al contempo. Scriveva:

Sulla base dei dati esegetici, dobbiamo riconoscere che la rivelazione biblica sul Consolatore stesso, come persona, è inesistente; come del resto non conosciamo la sua rivelazione ipostatica nella storia della Chiesa. Il Paraclito, personalmente, non è stato ancora manifestato, benché sia stato inviato alla Chiesa nei suoi doni neotestamentari della pentecoste (BULGAKOV, 2012, p. 288).

Lo Spirito Santo rivela l'altro, rivela il Figlio ed il Padre e rivela tutto ciò che è del Figlio ed è del Padre. Per questo il Figlio dopo la risurrezione annunciò che egli doveva ascendere, perché l'Altro Consolatore potesse giungere. Il Cristo ascende al Padre affinché lo Spirito discenda in persona. Solo in tal maniera la volontà del Padre si sarebbe potuta compiere attraverso il Figlio. È così che lo Spirito compie la volontà del Padre "“come se fosse suo proprio volere" (S. Simeone Nuovo Teologo). Nello stesso tempo egli ci "consola" dell'assenza visibile di Cristo. Il termine Paraclitos significa "colui che è chiamato presso", colui che si tiene "presso di noi" come nostro difensore, avvocato, testimone della nostra salvezza attraverso Cristo» (EVDOKIMOV, 1971, p. 116).

Vi è quindi un procedere unitario del mistero della salvezza dal Padre che è cristologico e pneumatologico insieme nella prospettiva teologica che stiamo esaminando. Perché il Cristo e lo Spirito sono inseparabili.

Seguendo i Padri si può anche dire che Cristo è il grande Precursore dello Spirito Santo. S. Atanasio afferma: "Il Verbo ha assunto la carne affinché noi possiamo 
ricevere lo Spirito Santo; Dio si è fatto sarcoforo affinché l'uomo possa divenire pneumatoforo (Atanasio, De Incarnatione, 8) [...] l'Ascensione del Cristo è l'epiclesi per eccellenza, poiché divina; il Figlio domanda al Padre di donare lo Spirito Santo, ed in risposta a questa supplica il Padre invia lo Spirito e fa erompere la Pentecoste (EVDOKIMOV, 1971, p. 117).

È una immagine meravigliosa quella che qui ci dona Evdokimov che ci rimanda al senso Liturgico della vita dell'uomo che sta in Cristo e vive adombrato dallo Spirito Santo perché il Padre possa dire: costui è figlio, è l'amato: «Tu sei Figlio mio» (Lc 3,22). Si rimarca l'inseparabilità del Figlio e dello Spirito anche nella vita dell'uomo redento. Questo aspetto si vede già negli eventi della salvezza - risurrezione, ascensione, pentecoste - ed anche si coglie una inseparabilità tra questi e ciò che è atteso per il compimento della salvezza, infatti pentecoste e parusia sono congiunte. Senza la grande pentecoste e la piccola pentecoste, quella personale, quella della conversione alla vita filiale ricevuta nel battesimo, ciò non sarebbe possibile e l'uomo non potrebbe vivere da figlio, accogliere cioè la propria autentica condizione creaturale.

La colomba che ha riposato sul Figlio al Giordano ora riposa su tutti coloro che sono stati costituiti figli e che stanno vivendo secondo l'ordine della redenzione, cioè al modo di Dio. Tutta la creazione protende verso quella pienezza ed attende ( $\mathrm{Rm} 8,19 \mathrm{ss}$ ) che si rinnovi in modo definitivo la sua condizione iniziale. Per questo la Chiesa è dinamizzata dallo Spirito, abbellita di tutti i suoi doni, per essere pronta per il suo Sposo: si può infatti dire che noi andiamo come assetati ad abbeverarci al costato e «abbeverati dello Spirito noi beviamo Cristo» (ATANASIO, PG 26, 576), ci nutriamo di lui.

È perciò indispensabile intendere la parusia non solo in relazione al Cristo, ma anche in relazione alla diade del Figlio e dello Spirito Santo, e quindi a tutta la Santa Trinità. La parusia quale manifestazione della gloria è simultaneamente una rivelazione sensibile dello Spirito Santo, riposante sul Figlio e dimorante nel mondo. La parusia del Cristo è, in questo senso, altresì la parusia dello Spirito Santo, la quale, se ha inizio a partire dalla pentecoste, sensibilmente però si compie in modo simultaneo a quella del Cristo (BULGAKOV, 1991, p. 579).

Lo Spirito Santo nel compiersi della storia della salvezza attualizza ciò che è proprio del procedere della pericoresi trinitaria che è un perenne dono e accoglienza: donarsi e riceversi.

Ciascuna persona divina realizza il proprio essere estatico nell'altra persona, nella relazione per questo ciascuna si compie nell'essere dell'altra. Così come il Padre, il Figlio e lo Spirito Santo sono un essere nell'altro, così l'amore, che è dare e riceversi reciproco, è partecipato all'uomo (Gv 17,21; Rm 5,1-5). 


\section{Lo Spirito agisce all'interno dell'uomo e ci rende dinamici dell'Amore relazionale}

Il vangelo di Giovanni bene esprime l'identità dell'uomo nuovo abitato dal Soffio di Dio (cfr. Gv 20,19-31), dell'uomo destinato a testimoniare il Risorto, chiamato a gioire dei beni di Dio Padre; è colui che aspira alla pienezza della sua gloria, pregustando già la gioia futura. Il Soffio è quindi l'organo

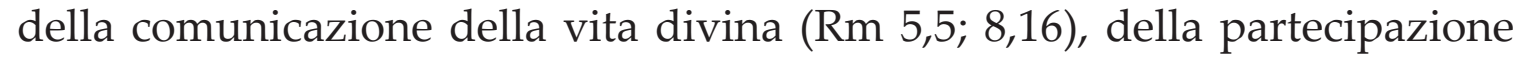
alla condizione dei figli (EVDOKIMOV, 1981b, p. 88).

Per Evdokimov l'azione dello Spirito Santo non è comprensibile con una analisi esclusivamente oggettiva dell'agire di Dio in relazione all'uomo e del suo partecipare se stesso. L'uomo vi coglierebbe altrimenti solo la difficoltà del combattimento incessante, della lotta. Mentre si tratta dell'accoglienza dei doni dello Spirito, si tratta di un duplice movimento che sospinge verso Dio e che ricolma dei suoi doni. L'uomo vive prima una fase di conversione, cioè di superamento dei propri limiti, di lotta, a cui segue un movimento di ascesi che scaturisce dall'aver accolto i doni dello Spirito Santo. Il quale, santificandoci, ci trasmette qualcosa della sua propria natura senza confusione $^{14}$, perché noi si possa compiere la volontà del Padre.

Lo Spirito Santo è $\pi \alpha v \gamma\llcorner o v$, santità ipostatizzata, non per appropriazione, ma per la sua natura stessa; "è la qualità della santità divina", precisa Cirillo Alessandrino; è il principio stesso della santificazione, che raggiunge immediatamente la creatura. Questa non possiede alcun dono che non venga dallo Spirito (san Basilio); lo Spirito è sostanzialmente presente nell'anima santificata (san Cirillo). Spirito Donatore, esso viene all'uomo e ne fa "dono al Padre". L'opera è così concepita dal Padre, eseguita dal Figlio e compiuta dallo Spirito Santo. Lo Spirito ricrea l'essere umano e lo rifà cristoforo, simile a Cristo, perché lo ha fatto prima pneumatoforo (EVDOKIMOV, 1981b, p. 398).

Lo Spirito Santo imprime nell'uomo il suo Soffio vitale e, senza confusione, lo Spirito Santo si fa co-soggetto della nostra vita in Cristo. L'uomo che è stato ricreato dallo Spirito, che è stato risoffiato dal Figlio del suo stesso Spirito porta Cristo al mondo, porta la sua immagine filiale e respira del suo stesso soffio, della sua stessa vita.

\footnotetext{
${ }^{14}$ Lo Spirito ci è donato come anticipazione di ciò che sarà pienamente alla fine dei tempi, quando Dio sarà in tutti. Si tratta di una presenza intima, irradiante che non dissolve le persone. Su questo aspetto la teologia cristiana converge nella riflessione delle diverse tradizioni. «La tradizione orientale insiste in modo particolare su questo aspetto della caparra del Regno e sulla sua manifestazione come luce: S. Gregorio Palamas, Serafino di Sarov. Riconosce inoltre un segno dell'inabitazione dei corpi santificati e dei miracoli che Dio opera vicino a questi corpi e per mezzo di essi [...] anche qui (in occidente) vengono attestati i riflessi sul corpo, la trasfigurazione del volto, l'irradiamento o le visioni di luce»: (Congar, 1981, p. 285-286). La teologia latina afferma che quando una delle Persona divine è posseduta dall'uomo, allora procura un dono, quello della gloria e del godimento perfetto. Lascia, insieme alla consolazione una "certa impressione di se stessa" e dei doni attraverso i quali godiamo della presenza ricevuta (TOMMASO D'AQUINO, I Sent., d.14, q.2, a.2, ad 2).
} 
L'azione santificante dello Spirito Santo precede ogni atto in cui lo spirituale si manifesta, prende corpo, si incarna nella storia e diventa manifestazione di Cristo (EVDOKIMOV, 1971, p. 113). Questo modo di agire dello Spirito Santo è coerente in tutta la storia della salvezza in cui si manifesta: nella creazione cova le acque (Gen 1,1), nell'annuncio adombra la Madre Vergine che diviene Madre di Dio (Lc 1,35), nel Battesimo sosta sul Figlio (Lc 3,22), l'unge e ne fa l'Unto il Cristo; nella pentecoste scende sui discepoli (Gv 20, 22-23) e li costituisce in un solo corpo (At 2,21), la chiesa ${ }^{15}$.

Ciò che Evdokimov descrive della storia della salvezza vede parallelamente nell'adesione dell'uomo a Dio: lo Spirito Santo è dato agli uomini nel Sacramento di rigenerazione che è il Battesimo, il regno di Dio non è attesa, ma è una realtà vivente nel cuore dell'uomo, che è abitato da Dio. Lo Spirito grida: Abbà, Padre! Per questo possiamo dire che lo Spirito è più intimo a noi che noi stessi, perché in noi scrive l'immagine del Figlio.

Se san Giovanni dice: "Noi saremo simili a lui" (1Gv 3,2), san Paolo da parte sua parla al presente: "Noi riflettiamo come in uno specchio la gloria del Signore, siamo trasformati in quella medesima immagine, sempre più gloriosa" (2Cor 3,18). Nel volto svelato i cristiani riflettono come Mosè la gloria del Cristo. La contemplazione di Dio nel Cristo li rende simili a Dio. Perciò la vita cristiana comporta la grazia di una certa visione di Dio, sia pure crepuscolare, che trasforma a sua immagine; si tratta di una visione che fa credere, conoscere, unirsi e trasformarsi come sua immagine e somiglianza (EVDOKIMOV, 2015, p. 40).

Pneumatizzazione, la chiama Evdokimov: «pneumatizzazione dell'essere umano mediante l'azione trinitaria: è l'ultimo grado della comunione tra il Dio uno e trino e l'uomo, "la conversione dell'anima a Dio", pur sempre conversione in energia e non in essenza. L'unione nella theosis è [...] energetica, senza che l'essenza umana divenga per questo l'essenza di Dio» (EVDOKIMOV, 1954-1955, p. 176). La natura umana è trasfigurata. La trasfigurazione della natura umana, tema centrale della teologia dell'Oriente cristiano, si realizza per l'azione dello Spirito che vivifica l'uomo e lo muove ad una adesione piena a Cristo fino a poter dire con l'apostolo: «Non sono più io che vivo, ma Cristo vive in me» (Gal 2,20), il Cristo si manifesta per la forza dello Spirito attraverso l'uomo che è testimone, portatore di Cristo, abitato dallo Spirito.

\section{Conclusione}

Abbiamo in questi brevi passaggi compreso come nella teologia di Evdokimov ciò che si è contemplato nell'epifania del Battesimo di Gesù sia espressione della condizione della vita dell'uomo redento che vive in

15 «La Chiesa è corpo: ciò significa che i membri della Chiesa costituiscono una unità organica - come le membra del corpo di carne - una unità di vita»: (YANNARAS, 1995, p. 41-42). 
Cristo adombrato dallo Spirito ${ }^{16}$, continuamente sospinto verso l'amore ardente che fa superare la prova della tentazione e guida fino all'ingresso nel regno: i battezzati sono sospinti di gloria in gloria, di grazia in grazia, di dono in dono, fino alla pienezza della loro condizione filiale.

Per l'uomo, infatti, sono state pronunziate tutte le parole divine e per lui sono stati compiuti i misteri della rivelazione. Tutto è stato fatto perché voi diveniate come altrettanti soli cioè forza vitale per gli altri uomini. Siate luci perfette dinanzi a quella luce immensa. Sarete inondati del suo splendore soprannaturale. Giungerà a voi, limpidissima e diretta, la luce della Trinità, della quale finora non avete ricevuto che un solo raggio, proveniente dal Dio unico, attraverso Cristo Gesù nostro Signore, al quale vadano gloria e potenza nei secoli dei secoli (GREGORIO NAZIANZENO, PG 36, p. 358-359).

Il messaggio di salvezza si innalza nella Chiesa che benedice tutta la creazione. Nulla è estraneo a Cristo, alla sua umanità, non vi è nulla che non abbia ricevuto l'unzione dello Spirito Santo, lo annuncia l'edificio stesso, in cui la Chiesa si riflette nelle icone dei suoi Santi. «ll piano sacramentale che spiega questa intimità profonda tra il cosmo e l'uomo: in esso la materia cosmica diviene mezzo conduttore, veicolo della grazia, delle energie divine. Tutto è unito nella Liturgia cosmica, tutto è sospeso al santo per ritrovarsi in esso e cantare un inno meraviglioso al suo Creatore» (EVDOKIMOV, 1981b, p. 120-121.). Lo Spirito vivente nell'uomo può cantare al Padre. Soltanto così il Cristo si manifesta in lui. Essere pneumatoforo per il dono sacramentale dello Spirito fa vivere da cristofori: ripieni dello Spirito Santo e suoi testimoni nel mondo, manifestatori di Cristo, cristofanici.

La proposta antropologica di Evdokimov si apre quando egli parla della chiesa che introduce gratuitamente nella dimensione del tempo e dello spazio liturgici, in essa adunati i cristiani si contemplano reciprocamente nella luce della gloria del cielo. In questa armonia la chiesa canta la comunione. La Liturgia cristiana è teocentrica, essa è tesa come memoriale liturgico verso la memoria divina, per questo il grido che si innalza è una invocazione incessante: «Lo Spirito e la Sposa dicono "Vieni Signore Gesù!"»(Ap 22, 16-21).

È il sospiro silente della Sposa, spinta a questa invocazione dallo Spirito Santo, nella certezza della venuta futura quando il seme del Regno di Dio,

\footnotetext{
16 "'Sei diventata bella avvicinandoti alla mia luce; avvicinandoti hai attirato su di te la partecipazione della mia bellezza... Essendosi avvicinata alla luce, l'anima diviene luce" (san Gregorio di Nissa) [...] L'apofatismo orientale rende testimonianza allo Spirito Santo. Persona che rimane sconosciuta ma che manifesta tutto di Dio e rende reale la vita spirituale. Quest'ultima, sempre cosciente, approfondisce la conoscenza spirituale che sant'Isacco chiama "il senso della vita eterna" $\mathrm{e}$ "il senso delle realtà nascoste". La sua perfezione è la contemplazione-partecipazione della luce divina della Santa Trinità, manifestata nella visione della Persona del Cristo trasfigurato, donata fin d'ora ai santi che vivono già la festa dell'incontro»: (EVDOKIMOV, 1973, p. 53).
} 
cresciuto in albero, avrà raggiunto la sua pienezza e la maturità perfetta. L'uomo nuovo abitato dallo Spirito Santo vive nella Liturgia della Chiesa una anticipazione del compimento e in essa egli si manifesta sempre più luminoso della luce divina che lo rivela figlio di Dio.

L'iniziativa divina domina il culto dall'inizio alla fine e fa comprendere che lo schema del culto, l'ordine liturgico non sono indifferenti. [...] La Liturgia dei catecumeni è la liturgia della Parola; l'Evangelo è posto al centro sul tavolo dell'altare; la lettura dell'apostolo (lo schaliach, l'apostolos di un uomo è come un altro se stesso) precede la lettura dell'Evangelo, seguita dalla predicazione. La Liturgia dei fedeli è la liturgia dell'Eucarestia, al suo centro vi è il calice. Si vede quanto sia impensabile una separazione o una opposizione. "Dio parla e la cosa è", ciò che la Parola ha annunziato, si effettua immediatamente, si adempie nel calice che è la Parola compiuta. Ė la "Parola fatta carne" e la sua azione è immediata: trasmutazione dei fedeli in "sostanza del Re" (EVDOKIMOV, 1981b, p. 365).

\section{Riferimenti}

AGOSTINO DI IPPONA. Sermo, 186-187; PL 38, 1002-1003.

ARJAKOVSKY, A. La génération des penseurs religieux de l'émigration russe. Paris: L'Esprit e la Lettre, 2002.

ATANASIO. Oratio de Incarnatione Verbi, PG 25.

ATANASIO. Lettere a Serapione. Roma: Città Nuova, 1986.

BASILIO DI CESAREA. Sullo Spirito Santo 49. Roma: Città Nuova, 1993.

BERDJAEV, N. I compiti spirituali dell'emigrazione russa. Put', Paris: nr.1, p. 3-8, sett. 1925.

BULGAKOV, S. N. L'Agnello di Dio: il mistero del Verbo Incarnato. Roma: Città Nuova, 1990.

BULGAKOV, S. N. La Sposa dell'Agnello. Bologna: Dehoniane, 1991.

BULGAKOV, S. N. Il Paraclito. Bologna: Dehoniane, 2012.

CATTANEO, E. Introduzione. In: CATTANEO, E. (Ed.) Lettere a Serapione. Roma: Città Nuova, 1986. p. 7-34.

CIRILLO DI ALESSANDRIA. De Incarnatione Domini, PG 75.

CONGAR, Y.M.J. Credo nello Spirito Santo. Brescia: Queriniana, 1981.

EVDOKIMOV, P.N. De la nature et de la grâce dans la théologie de l'Orient. In: L. BEAUDUIN (Ed.). 1054-1954. L'église et les églises. Neuf siècles de douloureuse séparation entre l'Orient et Occident. Etude et travaux offerts à Dom Lambert Beauduin, II. Chevetogne: Chevetogne, 1954-55. p. 171-195.

EVDOKIMOV, P.N. Quelques jalons sur un chemin de vie. In: Semence d'unité. Tournai: Casterman, 1965. 
EVDOKIMOV, P.N. Lo Spirito Santo nella tradizione Ortodossa. Roma: Paoline, 1971.

EVDOKIMOV, P.N. L'amore folle di Dio. Ed. Cinisello Balsamo (Mi): San Paolo, $1973 / 2015$.

EVDOKIMOV, P.N. Le età della vita spirituale. Bologna: Dehoniane, 1981a.

EVDOKIMOV, P.N. L'Ortodossia. Bologna: Dehoniane, 1981b.

EVDOKIMOV, P.N. La vita trasfigurata in Cristo. Roma: Lipa, 2001.

FOURCADE, M., De Soloviev à Boulgakov: la réception catholique de l'orthodoxie russe en France. Contacts, Paris, n. 238, p. 118-145, Avril-Juin. 2012.

GIANAZZA, P.G. Paul Evdokimov cantore dello Spirito Santo. Roma: LAS, 1983. p. 60.

GIOVANNI CRISOSTOMO. In Epistolam ad Philippenses, Homilia VI-VII, PG 62.

GREGORIO NAZIANZENO. Discorsi, Discorso 39 per il Battesimo del Signore, 20, PG 36.

GREGORIO NAZIANZENO. Oratio Theologica II, 19-23, PG 36.

GUITTAT-NAUDIN, M. La Réception de Valdimir Soloviev en France. In: DE LAUBIER, P. (Ed.). Vladimir Soloviev, Jacques Maritain et le personnalisme chrétien. Paris: Parole et Silence. Presses universitaires de IPC, 2008. p. 75-98.

ILARIO DI POITIERS. De Trinitate, VIII, 45-46, PL 10.

LADARIA, L.F. Il Dio vivo e vero. Milano: Piemme, 2002.

MASSIMO IL CONFESSORE. Ambigua ad Iohannem, PG 91, XXXVIII.

PUTTI, A.M. Il difficile recupero dello Spirito. Roma: GBPress, 2016a.

PUTTI, A.M. kénosi. In: BORRIELLO, L. (Ed). Nuovo Dizionario di Mistica. Città del Vaticano: Editrice Vaticana, 2016b. p. 1143-1146.

TOMMASO D'AQUINO, Commento alle Sentenze di Pietro Lombardo. Bologna: Studio Domenicano, 1999. v. 1.

YANNARAS, C. Verità e unità della chiesa. Milano: CENS, 1995. p. 41-42.

Manoscritto presentato il 14 maggio 2020 e approvato il 10 agosto 2020.

Alberta Maria Putti ha conseguito sia la Licenza che il Dottorato, con valutazione Summa cum laude, in Teologia Dogmatica presso la Pontificia Università Gregoriana. Attualmente è Docente Incaricato Associato presso il dipartimento di Teologia Dogmatica della stessa Università, dove amministra corsi di metodo teologico, Pneumatologia e Teologia Trinitaria. Orcid.org/0000-0002-2105-8493. E-mail: amputti@gmail.com

Indirizzo: Pontificia Università Gregoriana

Piazza della Pilotta 400185 ROMA 